\title{
Ferroelectric order associated with ordered occupancy at the octahedral site of the inverse spinel structure of multiferroic $\mathrm{NiFe}_{2} \mathrm{O}_{4}$
}

\author{
J. K. Dey, ${ }^{1}$ A. Chatterjee, ${ }^{1}$ S. Majumdar, ${ }^{1}$ A.-C. Dippel, ${ }^{2}$ O. Gutowski, ${ }^{2}$ M. v. Zimmermann, ${ }^{2}$ and S. Giri ${ }^{1, *}$ \\ ${ }^{1}$ School of Physical Sciences, Indian Association for the Cultivation of Science, Jadavpur, Kolkata 700032, India \\ ${ }^{2}$ Deutsches Elektronen-Synchrotron DESY, Notkestrasse 85, 22607 Hamburg, Germany
}

(Received 4 December 2018; revised manuscript received 19 March 2019; published 15 April 2019)

\begin{abstract}
We report a ferroelectric order at $\sim 98 \mathrm{~K}$ for $\mathrm{NiFe}_{2} \mathrm{O}_{4}$, which carries an inverse spinel structure with a centrosymmetric $F d \overline{3} \mathrm{~m}$ structure at room temperature. The value of spontaneous electric polarization is considerably high as $\sim 0.29 \mu \mathrm{C} / \mathrm{cm}^{2}$ for $5 \mathrm{kV} / \mathrm{cm}$ poling field. The electric polarization decreases considerably $(\sim 17 \%)$ around the liquid-nitrogen temperature upon application of $50 \mathrm{kOe}$ field, proposing a significant magnetoelectric coupling. The synchrotron diffraction studies confirm a structural transition at $\sim 98 \mathrm{~K}$ to a noncentrosymmetric structure of $P 4_{1} 22$ space group. The occurrence of polar order is associated with an ordered occupancy of $\mathrm{Ni}$ and $\mathrm{Fe}$ atoms at the octahedral sites of the $P 4_{1} 22$ structure, instead of random occupancies at the octahedral site of the inverse spinel structure. The results propose that $\mathrm{NiFe}_{2} \mathrm{O}_{4}$ is a member in the type-II multiferroics.
\end{abstract}

DOI: 10.1103/PhysRevB.99.144412

\section{INTRODUCTION}

Nickel ferrite with the chemical formula $\mathrm{NiFe}_{2} \mathrm{O}_{4}$ (NFO) attracts special attention for its significantly similar physical properties with magnetite. Magnetite has been recognized as the oldest known and the most studied magnet in science, with diverse applications in many areas such as rechargeable batteries [1,2], magnetic recording [3], medicine, and biology [4,5], and continues to excite with its complex and intriguing fundamental properties [6-13]. Despite many similarities, investigations on NFO have been less attempted and the unfolded issues are yet to be explored.

Magnetite crystallizes in the inverse spinel structure $(F d \overline{3} m)$ at room temperature. The formal chemical formula of $\mathrm{Fe}_{3} \mathrm{O}_{4}$ can be represented as $\mathrm{Fe}_{\mathrm{Te}}^{3+}\left[\mathrm{Fe}^{2+} \mathrm{Fe}^{3+}\right]_{\mathrm{Oc}} \mathrm{O}_{4}^{2-}$, where one-third of $\mathrm{Fe}$ occupies the tetrahedral $(\mathrm{Te})$ site in the $\mathrm{Fe}^{3+}$ state, and the remaining $\mathrm{Fe}^{2+}$ and $\mathrm{Fe}^{3+}$ equally occupy the octahedral (Oc) site. Analogous to $\mathrm{Fe}_{3} \mathrm{O}_{4}$, NFO also carries the inverse spinel structure, where $\mathrm{Ni}^{2+}$ replaces the $\mathrm{Fe}^{2+}$ at the octahedral site in the $F d \overline{3} m$ structure. The inverse spinel structure of NFO is illustrated in Fig. 1, where the tetrahedral $(8 b)$ site is occupied by $\mathrm{Fe}^{3+}$ and the octahedral $(16 c)$ site is occupied by $\mathrm{Ni}^{2+}$ and $\mathrm{Fe}^{3+}$ with a $50 \%$ occupancy, as highlighted by the bicolor atoms in the figure. The figure also partially depicts that the octahedra are connected to each other and linkage of the octahedra with a tetrahedral unit through the $\mathrm{O}$ atoms. Similar to the ferrimagnetic order of magnetite at high temperature (860 K) [12], NFO also orders ferrimagnetically at $853 \mathrm{~K}$ [14]. The low-temperature Mössbauer study in an external magnetic field proposed a collinear magnetic structure of NFO [15]. The neutron-diffraction study also confirms the collinear ferrimagnetic order at room temperature [16,17]. Plenty of works were performed on the doping effect of NFO, which significantly influenced the magnetic,

*Corresponding author: sspsg2@iacs.res.in electronic, and structural properties [15,18-26]. Nanoscale properties of NFO were investigated, where the magnetic and dielectric properties were significantly influenced by the size effect and interparticle interactions [27-33] and films [34-36]. The spin-filtering effect has been proposed using the firstprinciples density functional theory for NFO [37,38], which was corroborated for NFO ultrathin films [39]. Raman studies were carried out on NFO and argued for the possible occurrence of the short-range order at the octahedral site, either in the tetragonal $P 4_{1} 22$ or $P 4_{3} 22$ symmetry at low temperature [40-42]. The proposed results are analogous to the symmetry lowering at the Verwey temperature for magnetite. Unlike adequate structural investigations on magnetite [7-11], the low-temperature structural studies of NFO are still lacking, which need to be probed.

Herein, we report the synchrotron diffraction studies over a wide temperature range of 10-300 $\mathrm{K}$ and the analysis of the diffraction data using Rietveld refinement confirms a structural transition to a noncentrosymmetric structure $\left(P 4_{1} 22\right)$ at $\sim 98 \mathrm{~K}$. The results further suggest that the random occupancies at the octahedral site of the inverse spinel structure no longer exist below $\sim 98 \mathrm{~K}$. Instead, an ordered occupancy of $\mathrm{Ni}$ and $\mathrm{Fe}$ is proposed. Intriguingly, the structural transition to a noncentrosymmetric structure is associated with the occurrence of a spontaneous ferroelectric (FE) order with $T_{\mathrm{FE}}$ close to $98 \mathrm{~K}$. The value of electric polarization $(P)$ is considerably high, $\sim 0.29 \mu \mathrm{C} / \mathrm{cm}^{2}$ for a $5 \mathrm{kV} / \mathrm{cm}$ poling field, which is comparable to the values of promising typeII multiferroics [42-46]. The ferroelectric order has been reported for a vanadate [47], few chromate oxides [48-54], few chromate sulfides [55-59], and a cobalt aluminate oxide [60], which had an ordered spinel structure in the paraelectric state. However, the multiferroic order has rarely been explored in a system involving an inverse spinel structure, except for the classic example of ferroelectric order in magnetite [61-69]. Current results are analogous to the observed FE order at low temperature for magnetite, although the origin of the FE order 


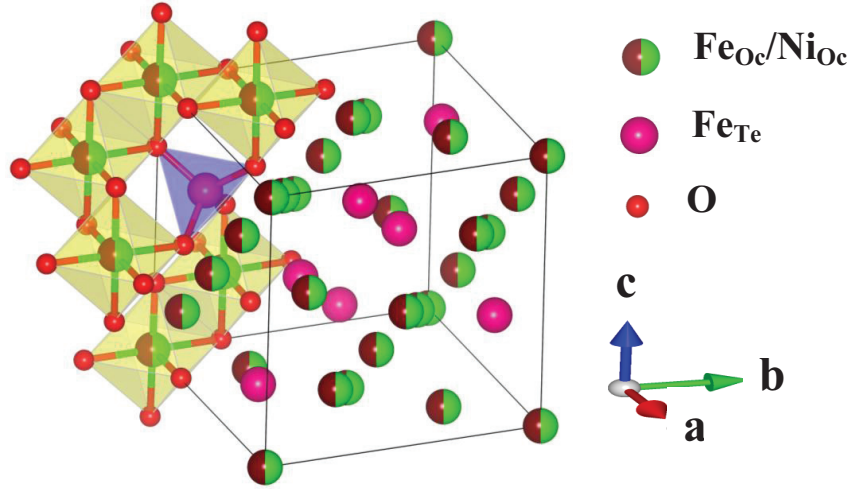

FIG. 1. Atomic positions in the inverse spinel structure of NFO. A portion of connecting $(\mathrm{Fe}, \mathrm{Ni}) \mathrm{O}_{6}$ octahedra around $\mathrm{FeO}_{4}$ tetrahedron is also depicted, where "Oc" and "Te" in the suffix indicate the octahedron and tetrahedron.

remains unsettled [61-69]. Here, the possible origin of the FE order in NFO is discussed based on the structural analysis.

\section{EXPERIMENTAL DETAILS}

Nickel ferrite with formula $\mathrm{NiFe}_{2} \mathrm{O}_{4}$ is prepared using the solid-state reaction [15]. The chemical composition is checked by the $\mathrm{x}$-ray diffraction studies at room temperature recorded in a PANalytical X-ray diffractometer (Model: X'Pert $\mathrm{PRO}$ ) using the $\mathrm{Cu} \mathrm{K} \alpha$ radiation. The single-phase chemical composition is further confirmed by the synchrotron x-ray diffraction studies recorded with a wavelength of $0.1422 \AA$ $(87.1 \mathrm{keV})$ at the P07 beam line of PETRA III, Hamburg, Germany, in the temperature range of 10-300 K. The synchrotron diffraction data are analyzed using the Rietveld refinement with a commercially available MAUD software. $\mathrm{X}$-ray photoemission spectroscopy (XPS) is recorded with a spectrometer of Omicron Nanotechnology. The powder sample pressed into a pellet is used for the dielectric measurements using an E4980A LCR meter (Agilent Technologies) equipped with a commercial PPMS Evercool-II system of Quantum Design. The pyroelectric current $\left(I_{p}\right)$ is recorded with an electrometer (Keithley, Model No. 6517B) at a constant temperature sweep rate. In all the measurements, the electrical contacts are fabricated using an air-drying silver paint. The poling electric fields are applied during cooling processes and $I_{p}$ measurements are carried out in the warming mode in the absence of an electric field. For the measurement of $I_{p}$, the electrical connections are short circuited before waiting for a sufficient period of time. Magnetization is measured with a commercial magnetometer of Quantum Design (MPMS, Evercool).

\section{EXPERIMENTAL RESULTS AND DISCUSSIONS}

The XPS measurements are carried out to detect the possible existence of $\mathrm{Fe}^{2+}$, which is attributed to the oxygen nonstoichiometry [70] or involving the doping effect [16]. Figure 2 depicts the Fe $2 p$ core-level spectrum of NFO, where the solid curve on the experimental data shows the satisfactory fit. The deconvolution of the peak into two components of

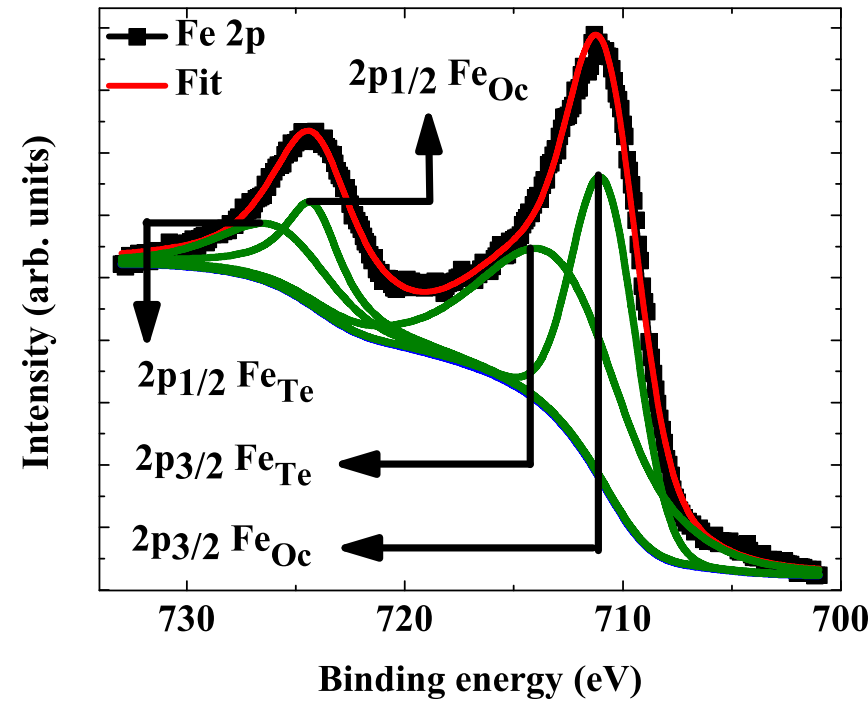

FIG. 2. Fe $2 p$ core-level XPS of NFO. Deconvolution of the curves along with the fit are shown by the solid curves.

$50 \%$ area under the curves correspond to the octahedral $2 p_{3 / 2}$ and $2 p_{1 / 2}$ peaks, and the tetrahedral $2 p_{3 / 2}$ and $2 p_{1 / 2}$ peaks. The peaks are observed at 712.9 and $710.8 \mathrm{eV}$ for tetrahedral Fe $2 p_{3 / 2}$ and octahedral Fe $2 p_{3 / 2}$, respectively, and at 725.8 and $724.2 \mathrm{eV}$ for tetrahedral $\mathrm{Fe} 2 p_{1 / 2}$ and octahedral Fe $2 p_{1 / 2}$, respectively. The results are consistent with the literature [70,71]. Here, the fit of the XPS spectrum indicates the absence of $\mathrm{Fe}^{2+}$ in NFO.

In order to observe the possible occurrence of the ferroelectric order in NFO, we first measure the dc-bias current $\left(I_{\mathrm{DC}}\right)$ using the bias electric field $(\mathrm{BE})$ method, as recently proposed to identify the genuine occurrence of ferroelectricity $[72,73]$. In the BE method, we record $I_{\mathrm{DC}}$ with a bias electric field of $5 \mathrm{kV} / \mathrm{cm}$ and a heating rate of $5 \mathrm{~K} / \mathrm{min}$ in the warming mode after cooling the sample down to $2 \mathrm{~K}$ in the zero electric field. The results of $\mathrm{BE}$ measurements in the temperature range of $2-120 \mathrm{~K}$ are depicted in Fig. 3(a). The inset of Fig. 3(a) highlights the magnified view around $98 \mathrm{~K}$, at which a clear signature of a "dip" is observed. In order to further confirm it, the pyroelectric current is recorded at different poling temperatures $\left(T_{\text {pole }}\right)$ for a $+5 \mathrm{kV} / \mathrm{cm}$ poling field. Here, the sample is always cooled from the selected $T_{\text {pole }}$ down to a low temperature (minimum up to $2 \mathrm{~K}$ ) and $I_{p}$ is measured during the warming mode in a zero field. The results of $I_{p}$ with $T$ at different $T_{\text {pole }}$ are depicted in Fig. 3(b) for a heating rate of $5 \mathrm{~K} / \mathrm{min}$. Here, different values of $T_{\text {pole }}$ are selected as representatives of the poling temperatures below, above, and close to $\sim 98 \mathrm{~K}$, at which a clear signature of a dip is observed in the BE method. In all of the cases, an apparent peak is always observed around $\sim 98 \mathrm{~K}$, as also depicted in the inset of the figure. The peak height around $98 \mathrm{~K}$ is nearly the same for $T_{\text {pole }} \geqslant 98 \mathrm{~K}$ (at 100 and $150 \mathrm{~K}$ ). The peak height decreases considerably for $T_{\text {pole }} \leqslant 98 \mathrm{~K}$ at $75,65,50$, and $30 \mathrm{~K}$, which is further highlighted in the inset of Fig. 3(b).

We further note that another peak is observed close to $T_{\text {pole }}$, as depicted in the inset of the figure for $T_{\text {pole }}$ below $\sim 98 \mathrm{~K}$. This peak does not engage with the phase transition at $98 \mathrm{~K}$, in accordance with the previous reports [73,74]. In addition 

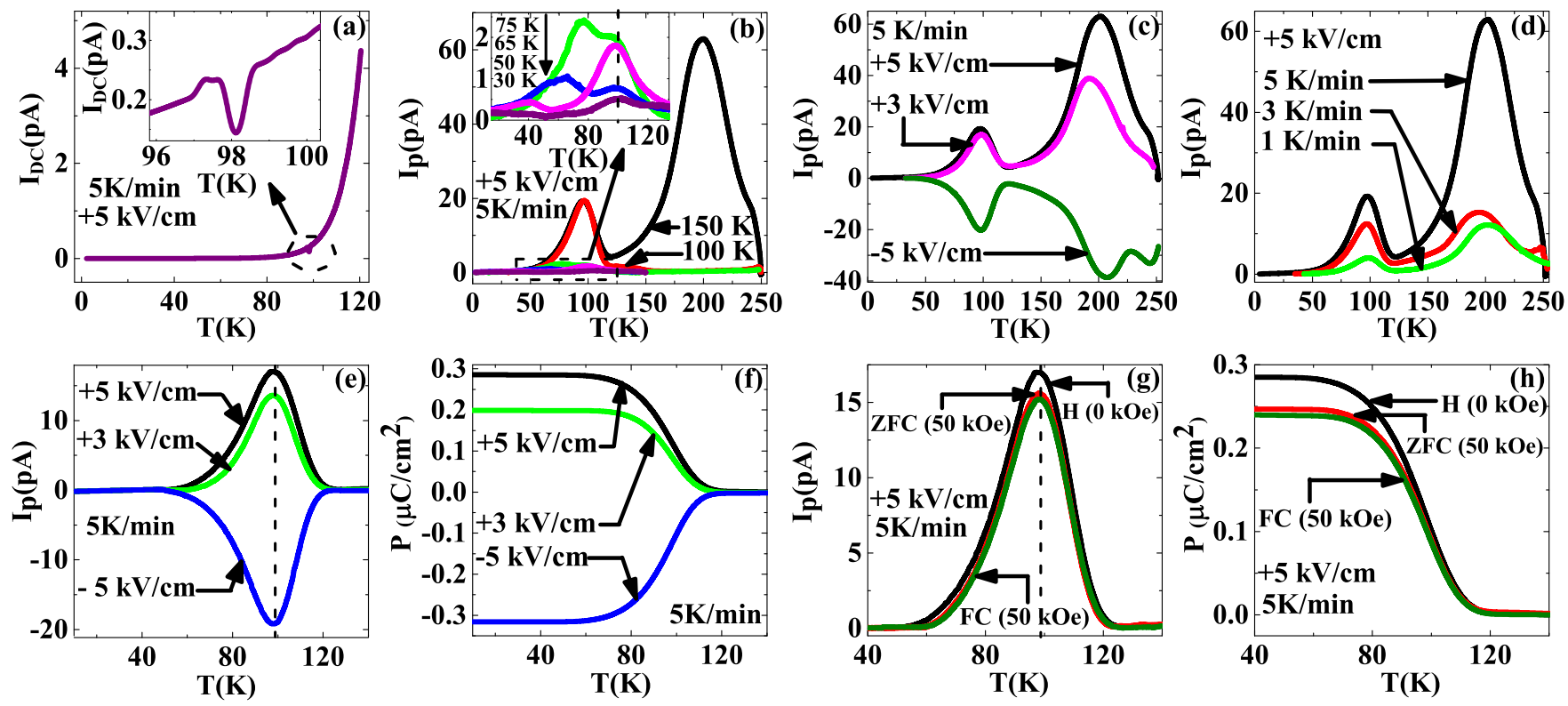

FIG. 3. (a) Temperature $(T)$ variation of dc-biased current $\left(I_{\mathrm{DC}}\right)$ with a $5 \mathrm{kV} / \mathrm{cm}$ bias field. The inset highlights the "dip" in $I_{\mathrm{DC}}(T)$ at $98 \mathrm{~K}$. $T$ variations of (b) pyroelectric current $\left(I_{P}\right)$ for different poling temperatures $\left(T_{\text {pole }}\right)$ at poling field $(E)$ of $5 \mathrm{kV} / \mathrm{cm}$, (c) $I_{P}$ for different $E$ and $T_{\text {pole }}=150 \mathrm{~K}$, (d) $I_{P}$ for different temperature sweep rates and $E=5 \mathrm{kV} / \mathrm{cm}$, (e) $I_{P}$ for different $E$ and $T_{\text {pole }}=150 \mathrm{~K}$ after subtracting the extrinsic thermally stimulated depolarization currents, (f) electric polarization $(P)$ at different $E$, (g) $I_{P}$, and (h) $P$ at $E=5 \mathrm{kV} / \mathrm{cm}$ in zero magnetic field and $50 \mathrm{kOe}$ using both the ZFC and FC modes. The inset of (b) highlights the peaks at the FE transition $\left(T_{C}\right)$. Vertical broken lines used in the inset of (b), (e), and (g) indicate the temperature at $T_{\mathrm{FE}}$.

to these peaks, another very large peak is observed around $\sim 200 \mathrm{~K}$, which occurs for $T_{\text {pole }}$ at $150 \mathrm{~K}$. The occurrence of a very large peak is rather consistent with those reported by Ngo et al. [74]. In the current investigation, the large peak around $200 \mathrm{~K}$ always appears for the measurement at different poling fields and heating rates, where $T_{\text {pole }}$ is considered to be $150 \mathrm{~K}$, as depicted in Figs. 3(c) and 3(d), respectively. This high-temperature large peak always appears when $T_{\text {pole }}$ is considered to be significantly larger than $T_{\mathrm{FE}}$. This large peak gets reversed for negative poling field, as depicted in Fig. 3(c). Importantly, this large peak disappears when $T_{\text {pole }}$ is $\leqslant \sim 98 \mathrm{~K}$, as shown in Fig. 3(b). In fact, this peak disappears for $T_{\text {pole }}=$ $100 \mathrm{~K}$, as depicted in Fig. 3(b), and confirms that the hightemperature large peak appears due to the extrinsic thermally stimulated depolarization currents (TSDCs) [73-76]. Thus, the evident signature of a peak around $\sim 98 \mathrm{~K}$ in both the pyroelectric current measurement and BE measurement confirms the genuine occurrence of the ferroelectricity with a $T_{\mathrm{FE}}$ at $98 \mathrm{~K}$.

Figure 3(e) depicts $I_{p}$ with $T$ for different poling fields $(E)$ and $T_{\text {pole }}=150 \mathrm{~K}$ after subtracting the high-temperature TSDC components. The large TSDC component is subtracted using the method suggested by Ngo et al. [74,75]. After subtracting the TSDC component, the $I_{p}(T)$ 's recorded at different heating rates are integrated over time, which nearly reproduce the same $P-T$ curve. The results indicate that the detrapped charges do not contribute to the $I_{p}$ values. Timeintegrated $I_{p}$ providing $P$ as a function of $T$ is depicted in Fig. 3(f) for different values of $E$. Reversal of $P$ due to a change in the sign of $E$ further signifies the ferroelectric behavior of NFO. The saturated value of $P$ in the current investigation is $\sim 0.29 \mu \mathrm{C} / \mathrm{cm}^{2}$ for $E=5 \mathrm{kV} / \mathrm{cm}$, which is significantly large compared to the values of the promising type-II multiferroics [42-46].

In order to probe possible magnetoelectric (ME) coupling, the effect of the magnetic field $(H)$ on $P(T)$ is investigated. The $I_{p}(T)$ is recorded with $H$ in both the zero-field-cooled (ZFC) and field-cooled (FC) modes [77]. In the case of the ZFC mode, the sample is cooled down to $\sim 10 \mathrm{~K}$ in the zero field and a magnetic field of $50 \mathrm{kOe}$ is applied in the warming mode during the measurement of $I_{p}$. For the FC mode, the sample is cooled in a field of $50 \mathrm{kOe}$ and $I_{p}$ is recorded during warming in a zero magnetic field. The results are summarized in Figs. 3(g) and 3(h). The results indicate an absence of noticeable change in the measurements for the ZFC and FC modes. However, a definite change is observed in the measurement of $H=50 \mathrm{kOe}$. We note that a significant decrease of $P$ is observed below $T_{\mathrm{FE}}$ due to $H$, as also shown in the figure, which indicates an existence of a magnetoelectric (ME) coupling. The percentage of decrease in $P$ is as considerable as $\sim 17 \%$ close to liquid-nitrogen temperature.

Magnetization $(M)$ recorded in the ZFC mode and dielectric permittivity $(\epsilon)$ at $H=0$ are measured with $T$. The values of $M$ and the real component $\left(\epsilon^{\prime}\right)$ of $\epsilon$ with $T$ are depicted around $T_{\mathrm{FE}}$ in Fig. 4(a), where a vertical broken line shows the position of temperature at $T_{\mathrm{FE}}$. In both the cases of $M(T)$ and $\epsilon^{\prime}(T)$, we could not detect any noticeable signature of $T_{\mathrm{FE}}$. The absence of the convincing signature in $\epsilon^{\prime}(T)$, close to $T_{\mathrm{FE}}$, has also been observed in other multiferroics [45,77-82]. The overlapping of the intrinsic component with the extrinsic contributions in $\epsilon$ such as the grain boundary and the sampleelectrode interface effects may lead to the weak signature or absence of any signature around $T_{\mathrm{FE}}$. Figure 4(b) depicts the magnetization curve, displaying a soft ferromagnetic 

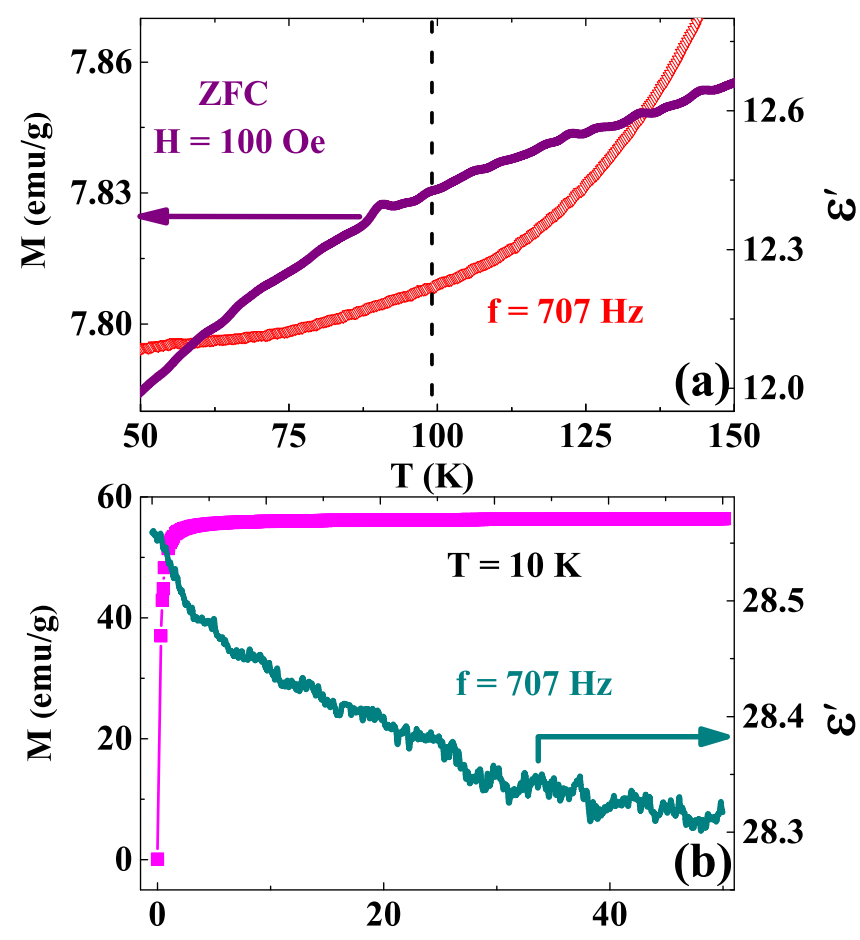

H (kOe)

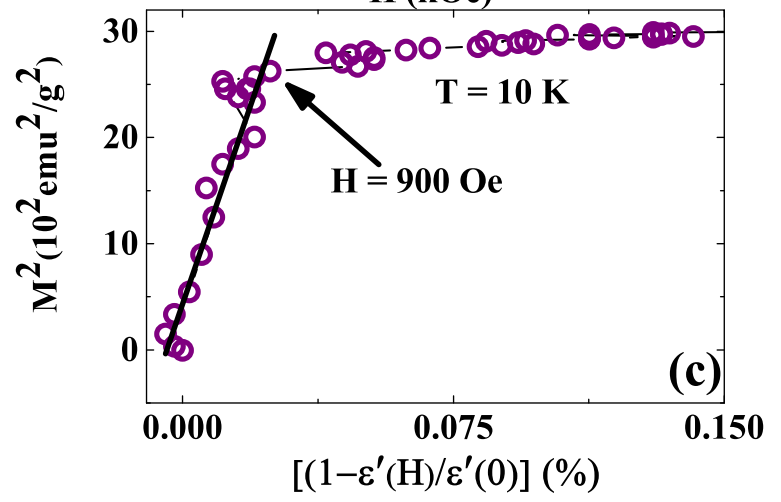

FIG. 4. Thermal variations of (a) ZFC magnetization ( $M$ ) (left axis) and real part $\left(\epsilon^{\prime}\right)$ of dielectric permittivity at frequency, $f=707 \mathrm{~Hz}$, (right axis) around $T_{\mathrm{FE}}$. Vertical broken line used in (a) indicates the temperature of $T_{\mathrm{FE}}$. Magnetic field $(H)$ dependence of (b) $M$ (left axis) and $\epsilon^{\prime}$ (right axis) at $10 \mathrm{~K}$. (c) Plot of $M^{2}$ with $\left[1-\epsilon^{\prime}(H) / \epsilon^{\prime}(0)\right](\%)$ at $10 \mathrm{~K}$. Straight line depicts the linear fit below 900 Oe.

character and variation $\epsilon^{\prime}$ with $H$ at $10 \mathrm{~K}$. At $10 \mathrm{~K}$, a small decrease of $\epsilon^{\prime}$ is observed with an increasing $H$. The percentage of magnetodielectric response, defined as $\left[1-\epsilon^{\prime}(H) / \epsilon^{\prime}(H=\right.$ $0)] \times 100$, is estimated to be $\sim 0.9 \%$ at $10 \mathrm{~K}$ for $H=50 \mathrm{kOe}$, which is comparable to the results for different multiferroics such as $\mathrm{CoCr}_{2} \mathrm{~S}_{4}$ [55], $M \mathrm{Cr}_{2} \mathrm{O}_{4}(M=\mathrm{Mn}, \mathrm{Co}, \mathrm{Ni})$ [83,84], $\mathrm{BiMnO}_{3}$ [85], $\mathrm{ZnCr}_{2} \mathrm{O}_{4}$ [86], $\mathrm{Sm}_{2} \mathrm{BaNiO}_{5}$ [45], and $R \mathrm{CrO}_{4}$ [82]. The magnetodielectric response is phenomenologically described by the Ginzburg-Landau theory for a second-order phase transition and is attributed to a ME coupling term $\gamma P^{2} M^{2}$ in the thermodynamic potential given by

$$
\Phi=\Phi_{0}+\alpha P^{2}-P E+\alpha^{\prime} M^{2}-M H+\gamma P^{2} M^{2} .
$$
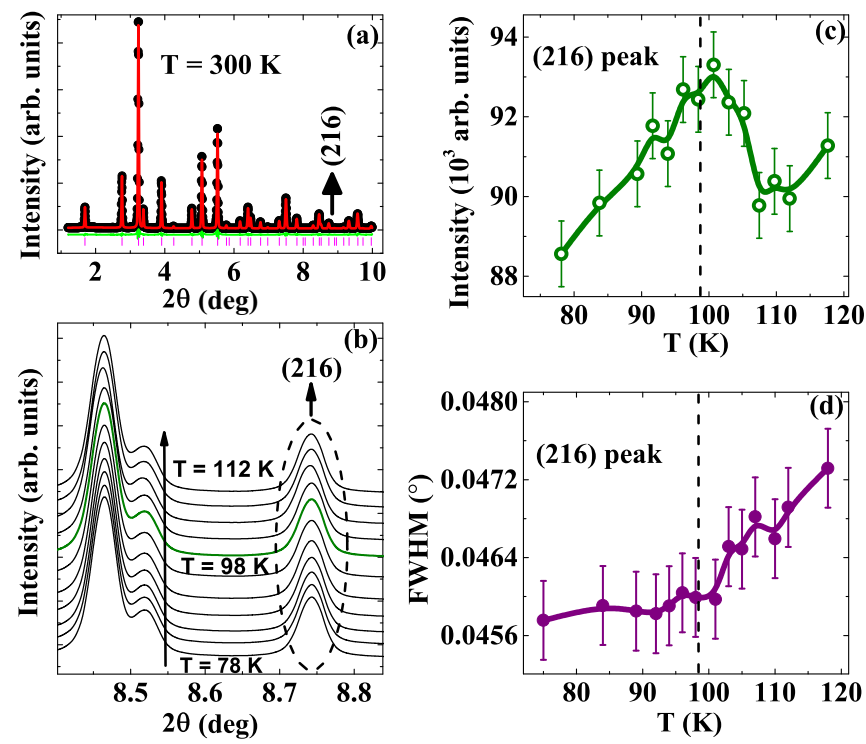

FIG. 5. Synchrotron diffraction patterns at (a) $300 \mathrm{~K}$ for NFO. Solid curve represents the fit using Rietveld refinement. (b) Selected diffraction peaks at different temperatures around $T_{\mathrm{FE}} . T$ dependence of (c) integrated intensities and (d) FWHM of the (216) peak. Vertical broken lines used in (c) and (d) indicate the temperature of $T_{\mathrm{FE}}$.

The $\alpha, \alpha^{\prime}$, and $\gamma$ in the above equation are the constants and functions of temperature. The linear dependence between squared magnetization and magnetodielectric response in the low-field regime indicate that the magnetodielectric coupling term $\gamma P^{2} M^{2}$ of the Ginzburg-Landau theory is significant. Here, a linear plot of $M^{2}$ with $\left[1-\epsilon^{\prime}(H) / \epsilon^{\prime}(H=0)\right] \times 100$ is observed below $\sim 0.9 \mathrm{kOe}$, as indicated by an arrow in Fig. 4(b), which has also been reported for the multiferroics with spinel structure $[55,83,84,86]$ and other multiferroic oxides $[45,82,85]$.

$\mathrm{NiFe}_{2} \mathrm{O}_{4}$ crystallizes in the inverse spinel structure with a centrosymmetric $F d \overline{3} m$ (No. $227, Z=8$ ) space group at $300 \mathrm{~K}$. At $300 \mathrm{~K}$, a satisfactory fit of the diffraction pattern using Rietveld refinement is depicted by the solid curve in Fig. 5(a). The refined positional coordinates are given in Table I considering the origin at the octahedral position. The reliability parameters, $R_{w}(\%) \sim 4.98, R_{\exp }(\%) \sim 3.04$, and $\chi^{2}(\%) \sim 1.76$ at $300 \mathrm{~K}$, are reasonable. In order to search for a possible structural change associated with the occurrence of a polar order, the selected diffraction peaks are plotted

TABLE I. Atomic positions of NFO with $F d \overline{3} m$ (No. $227, Z=8$ ) and $P 4_{1} 22$ (No. 91, $Z=4$ ) symmetries at 300 and $90 \mathrm{~K}$, respectively.

\begin{tabular}{ccccccc}
\hline \hline$T$ & Atoms & $x$ & $y$ & $z$ & Occupancy & site \\
\hline $300 \mathrm{~K}$ & $\mathrm{Ni}^{2+} / \mathrm{Fe}^{3+}$ & 0.00000 & 0.00000 & 0.00000 & $1.0(50 \%)$ & $16 c$ \\
& $\mathrm{Fe}^{3+}$ & 0.62500 & 0.62500 & 0.62500 & 1.0 & $8 b$ \\
& $\mathrm{O}^{2-}$ & 0.24797 & 0.24797 & 0.24797 & 1.0 & $32 e$ \\
$90 \mathrm{~K}$ & $\mathrm{Ni}^{2+}$ & 0.00000 & 0.75000 & 0.00000 & 0.5 & $4 a$ \\
& $\mathrm{Fe}^{3+}$ & 0.50000 & 0.75000 & 0.00000 & 0.5 & $4 b$ \\
& $\mathrm{Fe}^{3+}$ & 0.75000 & 0.75000 & 0.37500 & 1.0 & $4 c$ \\
& $\mathrm{O}^{2-}$ & 0.50792 & 0.75000 & 0.25000 & 1.0 & $8 d$ \\
& $\mathrm{O}^{2-}$ & 0.03935 & 0.25000 & 0.75000 & 1.0 & $8 d$ \\
\hline \hline
\end{tabular}



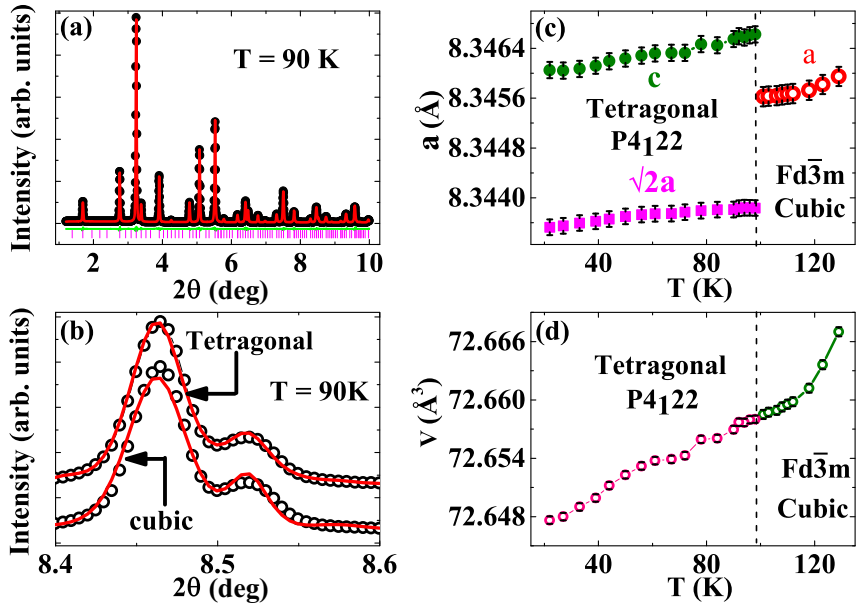

FIG. 6. Synchrotron diffraction patterns at (a) $90 \mathrm{~K}$ for NFO. Solid curve represents the fit using Rietveld refinement. Rietveld refinements are compared with (b) tetragonal $P 4_{1} 22$ and cubic $F d \overline{3} m$ space groups at $90 \mathrm{~K}$. $T$ dependence of (c) lattice constants and (d) unit-cell volume $(V)$. Vertical broken lines used in (c) and (d) indicate the temperature of $T_{\mathrm{FE}}$.

with temperature around $T_{\mathrm{FE}}$, as depicted in Fig. 5(b). The small changes around $T_{\mathrm{FE}}$ are noted, which are evident in the thermal variations of integrated intensity and full width at half maxima (FWHM) of the (216) diffraction peak, as depicted in Figs. 5(c) and 5(d), respectively. In Fig. 5(c), an apparent signature with a maximum is observed at $T_{\mathrm{FE}}$. This signature at $T_{\mathrm{FE}}$ may be associated with the change in the scattering cross section. Hence a change in the scattering amplitude may be correlated to this integrated intensity change and indicates the possible occurrence of a structural transition. The change in the integrated intensity is similar to that observed for the reported ferroelectric materials $[45,48,55,82]$. As depicted in Fig. 5(d), an anomaly in FWHM is also noted around $T_{\mathrm{FE}}$, where the decreasing trend in the thermal variation of FWHM becomes flat below $\sim T_{\mathrm{FE}}$.

Here we provide a low-temperature structural study for NFO. Recently, first-principles calculations proposed possible symmetry lowering to either $P 4_{1} 22$ (No. 91) or Imma (No. 74) space group at low temperature for NFO $[34,38,87,88]$. The Raman spectroscopy in epitaxial films as well as single crystal of NFO also proposed possible B site 1:1 ordering in the tetragonal $P 4_{1} 22$ symmetry [40,41]. A satisfactory refinement of the diffraction pattern at $90 \mathrm{~K}$ using the tetragonal $P 4_{1} 22$ structure with the higher symmetry in between $P 4_{1} 22$ (No. 91) or Imma (No. 74) space group, as proposed from the firstprinciples calculations $[34,38,87,88]$, is depicted by the solid curve in Fig. 6(a). The refined coordinates of the atoms are listed in Table I. The values of the reliability parameters, $R_{w}(\%) \sim 5.71, R_{\exp }(\%) \sim 3.04$, and $\chi^{2}(\%) \sim 1.87$ at $90 \mathrm{~K}$, are satisfactory. An example of the comparative fit of the selected diffraction peaks is highlighted in Fig. 6(b) using both the high-temperature $F d \overline{3} m$ structure and the proposed $P 4_{1} 22$ space group. The results clearly demonstrate the better fit of the diffraction peaks with the tetragonal $P 4_{1} 22$ space group and propose a structural transition to the tetragonal $P 4_{1} 22$ structure from the cubic $F d \overline{3} m$ structure.
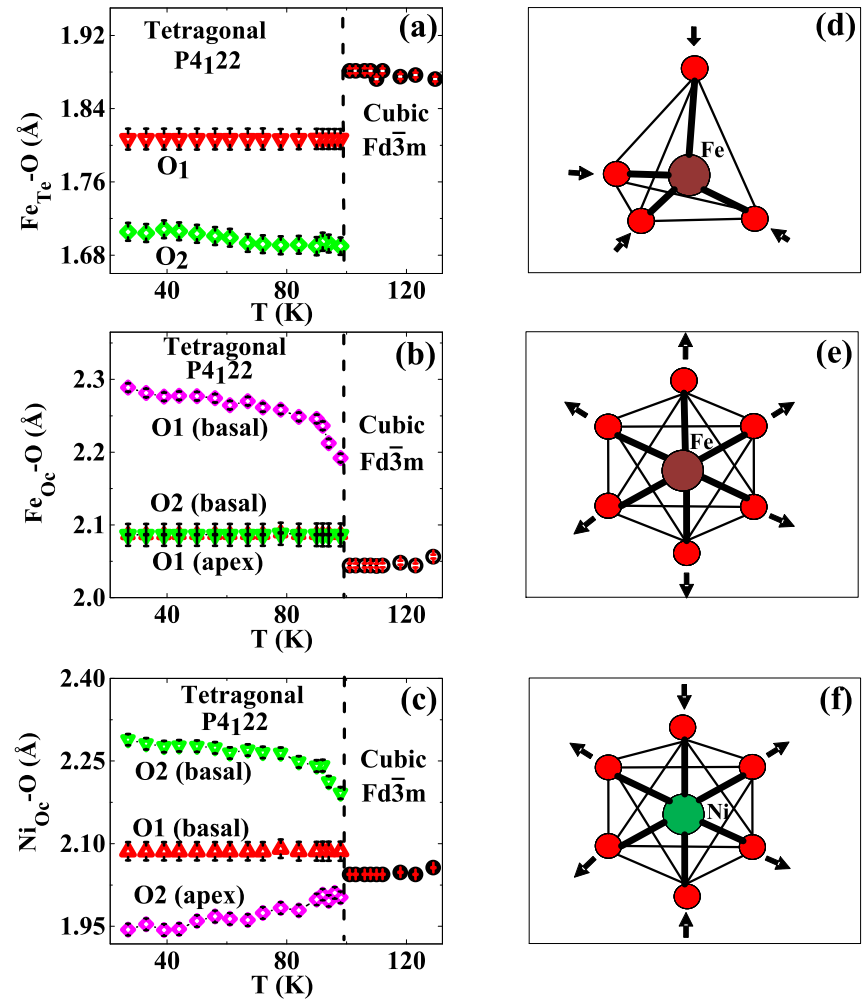

FIG. 7. Thermal variations of the bond lengths of (a) Fe tetrahedra $\left(\mathrm{Fe}_{\mathrm{Te}}-\mathrm{O}\right)$, (b) $\mathrm{Fe}$ octahedra $\left(\mathrm{Fe}_{\mathrm{Oc}}-\mathrm{O}\right)$, and (c) Ni octahedra $\left(\mathrm{Ni}_{\mathrm{Oc}}-\mathrm{O}\right)$. Vertical broken lines used in (a)-(c) indicate the temperature of $T_{\mathrm{FE}}$. Schematic representations of the distortions of the (d) $\mathrm{Fe}$ tetrahedron, (e) Fe octahedron, and (f) Ni octahedron.

Thermal $(T)$ variations of the lattice parameters are obtained from the refinement of the diffraction patterns at different temperatures. The $T$ variations of lattice constants are depicted in Fig. 6(c). Here, the $T_{\mathrm{FE}}$ in the figure is pointed out by the vertical broken line. A discontinuous change of lattice constants is observed at $T_{\mathrm{FE}}$. The $\sqrt{2} a$ and $c$ below the structural transition show a slow decrease with decreasing $T$. The results demonstrate that the ferroelectricity in NFO is correlated to this structural transition from the centrosymmetric $F d \overline{3} m$ to a noncentrosymmetric $P 4_{1} 22$ structure. The unit-cell volume $(V)$, as obtained from the lattice constants, is depicted in Fig. 6(d). Any convincing change in $V(T)$ is absent at $T_{\mathrm{FE}}$.

A significant structural distortion is noted at the structural transition. In order to understand these distortions microscopically, the bond lengths around the $\mathrm{Fe}$ and $\mathrm{Ni}$ atoms are estimated further. As given in Table I, the $P 4_{1} 22$ structure carries the two oxygen atoms, such as $\mathrm{O} 1$ and $\mathrm{O} 2$. Thus single $\mathrm{Fe}-\mathrm{O}$ bond length in the $F d \overline{3} m$ structure is converted to two bond lengths corresponding to $\mathrm{O} 1$ and $\mathrm{O} 2$, respectively, due to structural transition. In the $P 4_{1} 22$ structure, Fe atoms occupy the tetrahedral $(4 c)$ and octahedral $(4 b)$ sites. Four $\mathrm{Fe}-\mathrm{O}$ bond lengths are possible in the tetrahedral site. Thermal variations of two representative bond lengths corresponding to $\mathrm{O} 1$ and $\mathrm{O} 2$ are depicted in Fig. 7(a). The other two bond lengths behave similarly, which are not shown here. The magnitude of bond lengths decrease considerably at the structural transition, which decrease further with a further decrease in temperature 
and become temperature independent below the $T$ range of $\sim 70-80 \mathrm{~K}$. The decrease of bond lengths involving $\mathrm{O} 1$ and $\mathrm{O} 2$ are $\sim 4$ and $\sim 10 \%$, respectively, at $\sim 77 \mathrm{~K}$ compared to the value in the paraelectric state. A schematic representation of the distortion in the tetrahedral unit at the structural transition is shown in Fig. 7(d), as indicated by the arrows. Thus a contraction of the tetrahedral unit involves the structural transition.

The oxygen atoms involving the bond length along the crystallographic $c$ axis are designated as the apex oxygen atoms, with the remaining four being the basal oxygen atoms in the octahedral structure. Thermal variations of the $\mathrm{Fe}-\mathrm{O}$ bond lengths involving the basal and apex oxygen atoms are depicted in Fig. 7(b), when Fe atoms occupy the octahedral site. Here, the representative three bond lengths are shown in the figure. The three other remaining bond lengths behave similarly, which are not shown here. At the structural transition, the bond lengths increase discontinuously. With a further decreasing temperature, the bond length with apex oxygen remains nearly temperature independent, whereas bond lengths with basal oxygen atoms increase further and become temperature independent below the $T$ range of $\sim 70-80 \mathrm{~K}$. The increase of bond lengths at $\sim 77 \mathrm{~K}$ are $\sim 2, \sim 2$, and $\sim 10.5 \%$ with apex $\mathrm{O} 1$, basal $\mathrm{O} 2$, and basal $\mathrm{O} 1$ atoms, respectively. The schematic representation of the distortion of the octahedral unit at the structural transition is depicted in Fig. 7(e). Here, the arrows represent directions of the distortion at the structural transition and propose expansion of the Fe octahedral unit. Similar to the results of the Fe octahedral unit, the results of Ni octahedra are shown in Figs. 7(c) and 7(f). At the structural transition, the value of the $\mathrm{Ni}-\mathrm{O}$ bond length with apex $\mathrm{O} 2$ decreases, whereas the magnitude of the remaining two bond lengths involving basal oxygen atoms increases. The results propose a mixed distortion in the Ni octahedra, unlike the Fe octahedra. A contraction along the apex oxygen atoms and expansions of the octahedra with the basal oxygen atoms are noted, as depicted in Fig. 7(f). The decrease in bond lengths with apex $\mathrm{O} 2$ is $\sim 2 \%$ and increase in bond lengths with basal $\mathrm{O} 1$ and $\mathrm{O} 2$ are $\sim 2$ and $\sim 11 \%$, respectively.

The discover of the Verwey transition [7] in magnetite opened up many unexplored issues [1-13]. The possible charge ordering of $\mathrm{Fe}^{2+}$ and $\mathrm{Fe}^{3+}$ at the octahedral site, the nature of the structural transition, the occurrence of ferroelectricity, etc. are a few of those intricate issues, which still continue to attract fundamental interest. The charge ordering between $\mathrm{Fe}^{2+}$ and $\mathrm{Fe}^{3+}$ driven by the structural transition with ordered atomic positions at the octahedral sites has been correlated to the occurrence of ferroelectric order below the Verwey transition temperature of magnetite [89]. Analogous to that observed in magnetite, the occurrence of ferroelectricity in NFO is also correlated to the structural transition associated with an ordered arrangement of $\mathrm{Ni}$ and Fe atoms at the octahedral sites. The possible correlation of the charge ordering with the ferroelectric order in NFO attracts fundamental interest of the community. The results leave some unsolved issues, which need to be explored both theoretically and experimentally using sophisticated experimental tools. Although the preliminary ZFC magnetization as well as dielectricity do not indicate any convincing signature of ferroelectric order or structural transition, a reasonably strong magnetoelectric coupling is manifested by a $\sim 17 \%$ decrease of polarization upon application of $50 \mathrm{kOe}$ magnetic field close to the liquid-nitrogen temperature. Thus a delicate issue of possible modulation of the magnetic structure around ferroelectric order needs to be examined by incorporating the structural transition. Recently, Jong et al. proposed a ferroelectricity in NFO driven by the $p$ - $d$ hybridization from the first-principles study, where a much larger value of electric polarization of $23 \mu \mathrm{C} / \mathrm{cm}^{2}$ along the $z$ direction was estimated [88]. In the literature, the hybridization between the $3 d$ states of the $\mathrm{Fe}^{3+}$ cation and the $2 p$ states of oxygen induced by the Jahn-Teller effect was proposed [88]. Usually, the weak Jahn-Teller effect occurs in $\mathrm{Fe}^{3+}$ only in a low-spin state. Further theoretical and experimental studies are required to correlate the possible role of the Jahn-Teller effect on the appearance of ferroelectricity in NFO.

In conclusion, a FE order is observed for NFO at $\sim 98 \mathrm{~K}$ with a reasonably large value of the electric polarization of $\sim 0.29 \mu \mathrm{C} / \mathrm{cm}^{2}$ for a $5 \mathrm{kV} / \mathrm{cm}$ poling field. A considerable magnetoelectric coupling is confirmed by a $17 \%$ change of the electric polarization upon application of $50 \mathrm{kOe}$ magnetic field around $77 \mathrm{~K}$. The FE transition is associated with a structural transition to a noncentrosymmetric $P 4_{1} 22$ structure from the inverse spinel structure, as confirmed by the analysis of the synchrotron diffraction studies. The polar order is found to be associated with an ordered occupancy of the $\mathrm{Ni}$ and $\mathrm{Fe}$ atoms at the octahedral sites of the $P 4_{1} 22$ structure. The results suggest that NFO is a member of the type-II multiferroics. The results further propose that NFO demonstrates an analogous intricate scenario of magnetite and creates interest, which would lead to further investigations in other members of ferrites.

\section{ACKNOWLEDGMENTS}

S.G. acknowledges SERB, India project (Project No. SB/S2/CMP-029/2014) for the financial support. Portions of this work were carried out at the light source PETRA III of DESY, a member of the Helmholtz Association (HGF). Financial support (Proposal No. I-20170178) by the Department of Science and Technology (Government of India) provided within the framework of the India@DESY collaboration is gratefully acknowledged. J.K.D. and A.C. acknowledge DSTINSPIRE, India and UGC, India fellowships, respectively, for financial support.
[1] W. M. Zhang, X. L. Wu, J. S. Hu, Y. G. Guo, and L. J. Wan, Adv. Funct. Mater. 18, 3941 (2008).
[2] G. Zhou, D.-W. Wang, F. Li, L. Zhang, N. Li, Z.-S. Wu, L. Wen, G. Q. Lu, and H.-M. Cheng, Chem. Mater. 22, 5306 (2010). 
[3] D. Zhang, Z. Liu, S. Han, C. Li, B. Lei, M. P. Stewart, J. M. Tour, and C. Zhou, Nano Lett. 4, 2151 (2004).

[4] M. Ferrari, Nat. Rev. Cancer 5, 161 (2005).

[5] A. Jordan, R. Scholz, P. Wust, H. Fähling, and R. Felix, J. Magn. Magn. Mater. 201, 413 (1999).

[6] Z. Zhang and S. Satpathy, Phys. Rev. B 44, 13319 (1991).

[7] E. J. W. Verwey, Nature (London) 144, 327 (1939).

[8] M. Coey, Nature (London) 430, 155 (2004).

[9] J. P. Wright, J. P. Attfield, and P. G. Radaelli, Phys. Rev. B 66, 214422 (2002).

[10] J. M. Zuo, J. C. H. Spence, and W. Petuskey, Phys. Rev. B 42, 8451 (1990).

[11] M. S. Senn, J. P. Wright, and J. P. Attfield, Nature (London) 481, 173 (2012).

[12] K. Kato, S. Iida, K. Yanai, and K. Mizushima, J. Magn. Magn. Mater. 31-34, 783 (1983).

[13] B. A. Maher, I. A. M. Ahmed, V. Karloukovski, D. A. MacLaren, P. G. Foulds, D. Allsop, D. M. A. Mann, R. TorresJardón, and L. Calderon-Garciduenas, Proc. Natl. Acad. Sci. 113, 10797 (2016).

[14] S. Chikazumi, Physics of Ferromagnetism (Oxford University Press, New York, 1997).

[15] J. Chappert and R. B. Frankel, Phys. Rev. Lett. 19, 570 (1967).

[16] K. Ugendar, S. Samanta, S. Rayaprol, V. Siruguri, G. Markandeyulu, and B. R. K. Nanda, Phys. Rev. B 96, 035138 (2017).

[17] J. M. Hastings and L. M. Corliss, Rev. Mod. Phys. 25, 114 (1953)

[18] K. Kamala Bharathi, K. Balamurugan, P. N. Santhosh, M. Pattabiraman, and G. Markandeyulu, Phys. Rev. B 77, 172401 (2008).

[19] K. K. Bharathi, G. Markandeyulu, and C. V. Ramana, J. Phys. Chem. C 115, 554 (2011).

[20] N. Rezlescu and E. Rezlescu, Solid State Commun. 88, 139 (1993).

[21] N. Rezlescu, E. Rezlescu, C. Pasnicu, and M. L. Craus, J. Phys.: Condens. Matter 6, 5707 (1994).

[22] E. Rezlescu, N. Rezlescu, P. D. Popa, L. Rezlescu, and C. Pasnicu, Phys. Status Solidi A 162, 673 (1997).

[23] P. Parida, R. Kashikar, A. Jena, and B. R. K. Nanda, J. Phys. Chem. Solids 123, 133 (2018).

[24] K. Ugendar, V. R. Reddy, and G. Markandeyulu, IEEE Trans. Magn. 52, 1 (2016).

[25] A. Shan, X. Wu, J. Lu, C. Chen, and R. Wang, Cryst. Eng. Commun. 17, 1603 (2015).

[26] J. Sahariya, H. S. Mund, A. Sharma, A. Dashora, M. Itou, Y. Sakurai, and B. L. Ahuja, J. Magn. Magn. Mater. 360, 113 (2014).

[27] Z. Q. Wang, X. Y. Zhong, R. Yu, Z. Y. Cheng, and J. Zhu, Nat. Commun. 4, 1395 (2013).

[28] M. Rahimi, P. Kameli, M. Ranjbar, H. Hajihashemi, and H. Salamati, J. Mater. Sci. 48, 2969 (2013).

[29] M. Menelaou, K. Georgoula, K. Simeonidis, and C. DendrinouSamara, Dalton Trans. 43, 3626 (2014).

[30] Y. Wang, L. Li, Y. Zhang, X. Chen, S. Fang, and G. Li, J. Phys. Chem. C 121, 19467 (2017).

[31] V. Šepelák, I. Bergmann, A. Feldhoff, P. Heitjans, F. Krumeich, D. Menzel, F. J. Litterst, S. J. Campbell, and K. D. Becker, J. Phys. Chem. C 111, 5026 (2007).
[32] M. V. Ushakov, B. Senthilkumar, R. K. Selvan, I. Felner, and M. I. Oshtrakh, Mater. Chem. Phys. 202, 159 (2017).

[33] Z. Cveji, E. Duri, G. I. Ivandeki, B. Bajac, P. Postolache, L. Mitoseriu, V. V. Srdi, and S. Raki, J. Alloys Compd. 649, 1231 (2015).

[34] D. Fritsch and C. Ederer, Phys. Rev. B 82, 104117 (2010).

[35] F. Rigato, X. Martí, and J. Fontcuberta, J. Phys.: Conf. Ser. 303, 012013 (2011).

[36] U. Lüders, A. Barthélémy, M. Bibes, K. Bouzehouane, S. Fusil, E. Jacquet, J.-P. Contour, J.-F. Bobo, J. Fontcuberta, and A. Fert, Adv. Mater. 18, 1733 (2006).

[37] Z. Szotek, W. M. Temmerman, D. Ködderitzsch, A. Svane, L. Petit, and H. Winter, Phys. Rev. B 74, 174431 (2006).

[38] N. M. Caffrey, D. Fritsch, T. Archer, S. Sanvito, and C. Ederer, Phys. Rev. B 87, 024419 (2013).

[39] S. Matzen, J.-B. Moussy, P. Wei, C. Gatel, J. C. Cezar, M. A. Arrio, P. Sainctavit, and J. S. Moodera, Appl. Phys. Lett. 104, 182404 (2014).

[40] M. N. Iliev, D. Mazumdar, J. X. Ma, A. Gupta, F. Rigato, and J. Fontcuberta, Phys. Rev. B 83, 014108 (2011).

[41] V. G. Ivanov, M. V. Abrashev, M. N. Iliev, M. M. Gospodinov, J. Meen, and M. I. Aroyo, Phys. Rev. B 82, 024104 (2010).

[42] D. Khomskii, Physics 2, 20 (2009).

[43] K. Dey, A. Indra, A. Chatterjee, S. Majumdar, U. Rütt, O. Gutowski, M. V. Zimmermann, and S. Giri, Phys. Rev. B 96, 184428 (2017).

[44] J. Sannigrahi, S. Bhowal, S. Giri, S. Majumdar, and I. Dasgupta, Phys. Rev. B 91, 220407(R) (2015).

[45] A. Indra, K. Dey, S. Majumdar, I. Sarkar, S. Francoual, R. P. Giri, N. Khan, P. Mandal, and S. Giri, Phys. Rev. B 95, 094402 (2017).

[46] T. Kimura, Y. Sekio, H. Nakamura, T. Siegrist, and A. P. Ramirez, Nat. Mater. 7, 291 (2008).

[47] Q. Zhang, K. Singh, F. Guillou, C. Simon, Y. Breard, V. Caignaert, and V. Hardy, Phys. Rev. B 85, 054405 (2012).

[48] K. Dey, S. Majumdar, and S. Giri, Phys. Rev. B 90, 184424 (2014).

[49] K. Singh, A. Maignan, C. Simon, and C. Martin, Appl. Phys. Lett. 99, 172903 (2011).

[50] Y. Yamasaki, S. Miyasaka, Y. Kaneko, J.-P. He, T. Arima, and Y. Tokura, Phys. Rev. Lett. 96, 207204 (2006).

[51] Y. J. Choi, J. Okamoto, D. J. Huang, K. S. Chao, H. J. Lin, C. T. Chen, M. van Veenendaal, T. A. Kaplan, and S.-W. Cheong, Phys. Rev. Lett. 102, 067601 (2009).

[52] S. Yang, H. X. Bao, D. Z. Xue, C. Zhou, J. H. Gao, Y. Wang, J. Q. Wang, X. P. Song, Z. B. Sun, X. B. Ren, and K. Otsuka, J. Phys. D: Appl. Phys. 45, 265001 (2012).

[53] A. Maignan, C. Martin, K. Singh, C. Simon, O. I. Lebedev, and S. Turner, J. Solid State Chem. 195, 41 (2012).

[54] A. A. Bush, V. Y. Shkuratov, K. E. Kamentsev, A. S. Prokhorov, E. S. Zhukova, B. P. Gorshunov, and V. I. Torgashev, Phys. Rev. B 85, 214112 (2012).

[55] K. Dey, A. Karmakar, A. Indra, S. Majumdar, U. Rütt, O. Gutowski, M. V. Zimmermann, and S. Giri, Phys. Rev. B 92, 024401 (2015).

[56] J. Hemberger, P. Lunkenheimer, R. Fichtl, H.-A. Krug von Nidda, V. Tsurkan, and A. Loidl, Nature (London) 434, 364 (2005).

[57] G. Catalan and J. F. Scott, Nature (London) 448, E4 (2007). 
[58] S. Weber, P. Lunkenheimer, R. Fichtl, J. Hemberger, V. Tsurkan, and A. Loidl, Phys. Rev. Lett. 96, 157202 (2006).

[59] L. Lin, H. X. Zhu, X. M. Jiang, K. F. Wang, S. Dong, Z. B. Yan, Z. R. Yang, J. G. Wan, and J.-M. Liu, Sci. Rep. 4, 6530 (2015).

[60] S. Ghara, N. V. Ter-Oganessian, and A. Sundaresan, Phys. Rev. B 95, 094404 (2017).

[61] K. Kato and S. Iida, J. Phys. Soc. Jpn. 51, 1335 (1982).

[62] Y. Miyamoto, M. Kobayashi, and S. Chikazumi, J. Phys. Soc. Jpn. 55, 660 (1986).

[63] C. Medrano, M. Schlenker, J. Baruchel, J. Espeso, and Y. Miyamoto, Phys. Rev. B 59, 1185 (1999).

[64] M. Alexe, M. Ziese, D. Hesse, P. Esquinazi, K. Yamauchi, T. Fukushima, S. Picozzi, and U. Gösele, Adv. Mater. 21, 4452 (2009).

[65] R. Takahashi, H. Misumi, and M. Lippmaa, Phys. Rev. B 86, 144105 (2012).

[66] F. Schrettle, S. Krohns, P. Lunkenheimer, V. A. M. Brabers, and A. Loidl, Phys. Rev. B 83, 195109 (2011).

[67] M. Ziese, P. D. Esquinazi, D. Pantel, M. Alexe, N. M. Nemes, and M. Garcia-Hernández, J. Phys.: Condens. Matter 24, 086007 (2012).

[68] K. Dey, A. Ghosh, P. Modak, A. Indra, S. Majumdar, and S. Giri, Appl. Phys. Lett. 105, 142905 (2014).

[69] S. Mishra, K. Dey, U. Chowdhury, D. Bhattacharya, C. K. Ghosh, and S. Giri, AIP Adv. 7, 125015 (2017).

[70] G. Hassnain Jaffari, A. K. Rumaiz, J. C. Woicik, and S. Ismat Shah, J. Appl. Phys. 111, 093906 (2012).

[71] S. Hüfner, Photoelectron Spectroscopy - Principles and Applications (Springer-Verlag, Berlin, 1995).

[72] C. De, S. Ghara, and A. Sundaresan, Solid State Commun. 205, 61 (2015).

[73] N. Terada, Y. S. Glazkova, and A. A. Belik, Phys. Rev. B 93, 155127 (2016).
[74] T. N. M. Ngo, U. Adem, and T. T. M. Palstra, Appl. Phys. Lett. 106, 152904 (2015).

[75] R. Chen and Y. Kirsh, Analysis of Thermally Stimulated Process (Pergamon, New York, 1981).

[76] W. Liu and C. A. Randall, J. Am. Ceram. Soc. 91, 3245 (2008).

[77] A. Indra, K. Dey, A. Midya, P. Mandal, O. Gutowski, U. Rütt, S. Majumdar, and S. Giri, J. Phys.: Condens. Mater. 28, 166005 (2016).

[78] K. R. S. Preethi Maher, A. Wahl, A. Maignan, C. Martin, and O. I. Lebedev, Phys. Rev. B 89, 144401 (2014).

[79] A. Ghosh, A. Pal, K. Dey, S. Majumdar, and S. Giri, J. Mater. Chem. C 3, 4162 (2015)

[80] A. Ghosh, K. Dey, M. Chakraborty, S. Majumdar, and S. Giri, Europhys. Lett. 107, 47012 (2014).

[81] B. Rajeswaran, D. I. Khomskii, A. K. Zvezdin, C. N. R. Rao, and A. Sundaresan, Phys. Rev. B 86, 214409 (2012).

[82] A. Indra, K. Dey, J. K. Dey, S. Majumdar, U. Rütt, O. Gutowski, M. v. Zimmermann, and S. Giri, Phys. Rev. B 98, 014408 (2018).

[83] T. D. Sparks, M. C. Kemei, P. T. Barton, R. Seshadri, E.-D. Mun, and V. S. Zapf, Phys. Rev. B 89, 024405 (2014).

[84] N. Mufti, A. A. Nugroho, G. R. Blake, and T. T. M. Palstra, J. Phys.: Condens. Matter 22, 075902 (2010).

[85] T. Kimura, S. Kawamoto, I. Yamada, M. Azuma, M. Takano, and Y. Tokura, Phys. Rev. B 67, 180401(R) (2003).

[86] J. K. Dey, S. Majumdar, and S. Giri, J. Phys.: Condens. Matter 30, 235801 (2018).

[87] D. Fritsch and C. Ederer, Phys. Rev 86, 014406 (2012).

[88] U.-G. Jong, C.-J. Yu, Y.-S. Park, and C.-S. Ri, Phys. Lett. A 380, 3302 (2016).

[89] J. van den Brink and D. I. Khomskii, J. Phys.: Condens. Matter 20, 434217 (2008). 